\title{
Effect of slit length on linear and non-linear acoustic transfer impedance of a micro-slit plate
}

\author{
Alessia Aulitto $^{1, *}$ (D), Avraham Hirschberg ${ }^{2}$, Ines Lopez Arteaga ${ }^{1}$ (D), and Esmée L.R.H. Buijssen ${ }^{1}$ \\ ${ }^{1}$ Department of Mechanical Engineering, Dynamics and Control, Eindhoven University of Technology, P.O. Box 513, 5600 MB \\ Eindhoven, The Netherlands \\ ${ }^{2}$ Department of Applied Physics, Fluid and Flows, Eindhoven University of Technology, P.O. Box 513, 5600 MB Eindhoven, The \\ Netherlands
}

Received 26 July 2021, Accepted 20 December 2021

\begin{abstract}
The effect of the slit length on the acoustic transfer impedance of micro-slit plates (MSPs) is investigated in the linear and non-linear regime for a specific slit geometry. This geometry is inspired by slits obtained by cutting and bending the plate. MSPs are plates with arrays of slit-shaped perforations, with the width of the order of the acoustic viscous boundary layer thickness. Impedance tube measurements on two accurately manufactured plates are compared to numerical solution of the Linearized Navier-Stokes equations and to analytical limits. The impedance of the plate is obtained by the impedance of a single slit divided by the plate porosity. The resistance of a slit is independent on the slit length and on the plate porosity. In the linear regime the resistance is accurately predicted by a two-dimensional numerical model. In the non-linear regime, the resistance is strongly dependent on the amplitude of the acoustic waves. The inertance of the slit is weakly dependent on the slit length and on the plate porosity, for low and moderate amplitudes. For high amplitudes, a complicated amplitude dependency of the inertia of short slits is found. One expects that most of the conclusions obtained can be generalised to other slit geometries.
\end{abstract}

Keywords: Micro-slit, Plates, Impedance, Absorption

\section{Introduction}

Micro-slit absorbers and plates (MSAs, MSPs) have been proposed as sound absorbers at low frequencies, providing lightweight and compact solutions to substitute conventional materials [1]. MSPs are plates with slit widths in the sub-millimeter range and low porosity (order of $1 \%$ ). In conventional designs, micro-slit plates are backed by a cavity forming micro-slit absorbers (MSAs). One of the advantages of slits with respect to circular perforations is that, for equal porosity, a single slit replaces a large number of circular perforations. Furthermore, a slit can be used to delimit flexible structures whose vibration can contribute to the sound absorption $[2,3]$. However, the manufacturing of slits is difficult and can be an obstacle in industrial applications. A possible manufacturing process is to cut the plate, bending the two portions close to the cut, as displayed in Figure 1. A slit is created without removing material from the plate and can lead to new designs. One of the advantages of this geometry is that the edges in contact with the slits are protected from external agents in harsh environments. Another advantage is the possibility to reach sub-millimeter slit widths. This manufacturing technique

*Corresponding author: a. aulitto@tue.nl is used to produce ${ }^{\circledR}$ Acustimet plates by Sontech $[4,5]$. In this work, a geometry inspired by the geometry of Figure 1 is studied. Impedance tube measurements are used to investigate the effect of the slit length in two accurately manufactured micro-slit plates. The edges of the slits are kept as sharp as possible. Both plates have the same porosity and total slit perforation length. In the linear regime, experimental results are compared to numerical solutions of the Linearized incompressible Navier-Stokes equations. Microperforated plates (MPPs) and micro-slit plates can be designed to obtain excellent linear acoustic properties but, at high amplitudes, the non-linear effects deteriorate the performance of the absorbers [6-8]. In practical applications, the acoustic particle velocity in the slits can reach high amplitudes. For this reason, the change of resistance and inertance of the slits due to non-linear effects for long and short slits has been studied in this this work. In literature, several manufacturing techniques are employed to create slits. In classical applications, a slit can be created by removing material from the plate $[2,3,9]$. Slits can also be generated by mating two slotted layers $[6,10]$. Alternative designs of MSAs have been reported in the literature [2, $3,6,11,12$. Several publications concern the acoustic impedance of MPPs at high amplitudes in presence of a bias flow or for sound-excited flows [13-17]. In Section 2, the 


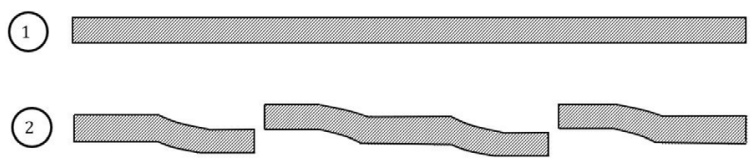

Figure 1. Representation of a typical geometry obtained with the process of cutting the plate and bending the extremities.

theoretical background is presented for the linear and nonlinear regimes. In Section 3, the experimental setup and the geometry of the plates are discussed. In Section 4, the twodimensional numerical model is described. In Section 5, the comparison between numerical and experimental results is presented in the linear and non-linear regimes. As explained by Cummings and Eversman [18], non-linear losses at very high amplitudes are due to the formation of a quasi-steady jet flow. This jet displays a contraction after flow separation from the edges of the slit: the so-called vena contracta. In Appendix A, the vena contracta factor for a simplified model of the geometry is calculated. In Appendix B, the quasi-steady incompressible model is presented. In Appendix C, a correction of the quasi-steady model for the viscous boundary layer thickness is discussed.

\section{Theoretical background}

In the linear regime, dissipation of acoustic energy takes place in the oscillating boundary layer of thickness $\delta_{v}=\sqrt{2 \mu / \omega \rho}$, where $\omega=2 \pi f$ is the angular frequency, $\rho$ is the air density $\left(\rho=1.18 \mathrm{~kg} / \mathrm{m}^{3}\right.$ at $25^{\circ} \mathrm{C}$ and atmospheric pressure) and $\mu$ is the dynamic viscosity of air $(\mu=1.85 \times$ $10^{-5} \mathrm{~kg} / \mathrm{ms}$ at $25^{\circ} \mathrm{C}$ ). The ratio between the slit width $b$ and the thickness of the viscous boundary layer $\delta_{v}$ is the Shear number

$$
\mathrm{Sh}_{b}=\frac{b}{\delta_{v}}
$$

In a micro-slit plate, typical Shear numbers, in the frequency range of interest, are of order unity. In the nonlinear regime, for moderate excitation amplitudes, vortices form locally at the edges of the slits. At very high amplitudes, this leads eventually to the formation of jets. Additional dissipation of acoustic energy is involved. The behaviour of the plate can be studied as a function of the Strouhal number $\left(\mathrm{St}_{b}\right)$, defined as the ratio between the slit width $b$ and the amplitude of the oscillating particle displacement at the slits. In formulas,

$$
\mathrm{St}_{b}=\frac{\omega b}{U_{p}},
$$

where $U_{p}$ is the cross-sectional surface averaged acoustic velocity amplitude at the slit $\left(u_{p}(t)=\operatorname{Re}\left[\hat{u}_{p} \exp (\mathrm{i} \omega t)\right]=\right.$ $U_{p} \cos (\omega t)$, for harmonic oscillations). For $\mathrm{St}_{b} \gg 1$ (linear regime) the particle displacement is smaller than the slit width and vortices are not formed. For $\mathrm{St}_{b} \ll 1$ (strongly non-linear), vortices are formed and they move away from the slit forming a free jet. For $\mathrm{St}_{b} \approx 1$ (moderate nonlinear) vortices form at the edges of the slits and they remain local. Alternatively, one can define a Strouhal number $\mathrm{St}_{t}$ based on the plate thickness at the slit $\left(t=t_{p}-t_{d}\right.$ in Fig. 3$)$ in order to compare the vortices displacement with the thickness of the plate at the slit [19].

\subsection{Transfer impedance in linear regime}

In the linear regime, the concept of transfer impedance is introduced in the frequency domain (for purely harmonic oscillations) of frequency $f$. At a distance large compared to the slit width $b$ but small compared to the acoustic wavelength $\lambda=c / f$, the flow can be described in terms of plane acoustic waves. As this region is compact, the corresponding complex amplitude $\hat{u}$ of the acoustic velocity $u(t)=\operatorname{Re}[\hat{u} \exp (\mathrm{i} \omega t)]=U \cos (\omega t)$ is the same on both sides of the plate. The transfer impedance of the plate is defined as the ratio between the complex acoustic pressure difference $\Delta \hat{p}$ and the amplitude of the acoustical velocity $\hat{u}$ in a cross-section upstream of the plate. The pressure is found by the extrapolation of the plane wave solutions (on both sides) to the sample surface (a formal discussion can be found in Sect. 4). The dimensionless transfer impedance of the plate is

$$
z_{\text {plate }}=\frac{\Delta \hat{p}}{\rho c \hat{u}},
$$

where $\rho$ is the density of air and $c$ is the speed of sound in air. Note that the plate transfer impedance is a complex quantity. The dimensionless transfer impedance of the plate $z_{\text {plate }}$ is

$$
z_{\text {plate }}=\operatorname{Re}\left[z_{\text {plate }}\right]+\operatorname{iIm}\left[z_{\text {plate }}\right],
$$

with $\mathrm{i}^{2}=-1, \operatorname{Re}\left[z_{\text {plate }}\right]$ the resistive part of the transfer impedance of the plate (or resistance of the plate) and $\operatorname{Im}\left[z_{\text {plate }}\right]$ the reactive part of the transfer impedance of the plate (or inertance of the plate). The transfer impedance of a slit is defined as the ratio between the complex pressure difference $\hat{p}$ and the amplitude of the crosssectional acoustical velocity $\hat{u}_{p}$ in the slit. In formulas,

$$
z_{\text {slit }}=\frac{\Delta \hat{p}}{\rho c \hat{u}_{p}}=z_{\text {plate }} \Phi,
$$

where $\hat{u}_{p}=\hat{u} / \Phi$ and $\Phi$ the porosity of the plate. Therefore, in the first order of approximation the transfer impedance of the plate can be obtained by the impedance of a single slit. In practical applications, the micro-slit plate is backed by a cavity. As the slit forms the neck of a Helmholtz resonator with a portion of the back cavity as volume, the flow within the slit will be considered as locally incompressible up to the first resonance frequency of the resonator. In the audio range, the square of the Helmholtz number is small, i.e. $\mathrm{He}^{2}=\left(\frac{\omega b}{c}\right)^{2}<10^{-1}$. Therefore, one can assume a frictionless flow of the incompressible flow. Thermal effects in the slit are neglected [20].

\subsection{Non-linear regime}

In the non-linear regime, the resistance due to vortex shedding dominates the absorption mechanism, as shown by Ingard and Ising [21]. Cummings and Eversmann [18] 
assume a quasi-steady flow behaviour to describe the behaviour of perforations at high Shear numbers and very high amplitudes of acoustic particle velocity. The model assumes that the acoustic flow separates at the edges of the slits and forms a free jet with a cross-section smaller than the perforation area. The ratio between the cross-section of the jet and the cross-section of the perforation is called the vena contracta factor $C_{v}$. In their model, using the Bernoulli equation one can derive the relationship between pressure change across the plate and particle velocity $\Delta \hat{p} \approx \frac{1}{2} \rho \hat{u}_{p}\left|\hat{u}_{p}\right|$ and $\hat{u}_{p}$, with $u_{p}(t)=\operatorname{Re}\left[\hat{u}_{p} \exp (\mathrm{i} \omega t)\right]=$ $U_{p} \cos (\omega t)$. For $\mathrm{St}_{b} \ll 1$ one can assume a quasi-steady incompressible flow with a free jet of vena contracta factor $C_{v}$. Assuming a harmonically oscillating velocity, $u(t)=U \cos (\omega t)$, one can calculate the time-averaged dissipated power and define the (time-averaged) non-linear dimensionless plate resistance Real $\left[z_{\text {plate,NL }}\right]$ as

$$
\operatorname{Real}\left[\mathrm{z}_{\mathrm{plate}, \mathrm{NL}}\right]-\operatorname{Real}\left[\mathrm{z}_{\mathrm{plate}, \mathrm{L}}\right]=\frac{4}{3 \pi} \frac{U}{C_{v}^{2} \Phi^{2} c},
$$

where Real $\left[z_{\text {plate,L}}\right]$ is the plate resistance in the linear regime. Derivation of the theoretical limit can be found in Appendix B. The vena contracta coefficient for the geometry discussed in this study is assumed to be $C_{v}=0.82$. This value is found in the potential flow limit for small porosity using the hodographic method [22, 23]. Derivation of the vena contracta factor $C_{v}$ can be found in Appendix A. The theoretical limit can be corrected for the effect of the quasi-steady viscous boundary layer. This causes a reduction of the effective porosity that leads to an increase of the resistance. The correction for the thickness of the viscous boundary layer is in Appendix C. For the inertial part of the transfer impedance $\operatorname{Im}\left[z_{\text {plate }}\right]$, Ingard and Ising assume that in the upstream of the flow separation the flow remains identical to the potential flow prevailing in the linear case [21]. In the downstream free jet, the inertia is negligible. Hence, the inertia should be reduced by a factor 2 . In other words, $\Delta \operatorname{Im}\left[z_{\text {plate, NL }}\right]=-\operatorname{Im}\left[z_{\text {plate, } \mathrm{L}}\right] / 2$. The factor onehalf is explicitly discussed in Morse and Ingard [24]. This simple limit will be compared to experimental results in Section 5.2. To study non-linear effects, the linear contribution is subtracted from the non-linear resistance and inertance. In order to analyze the effect on a single slit, the porosity $\Phi$ is introduced. The change in resistance (Real $\left.\left[z_{\text {plate,NL }}\right]-\operatorname{Real}\left[z_{\text {plate, }}\right]\right)$ is normalized with the non-linear limit proposed by Ingard and Ising [21] and corrected by the vena contracta factor as in Temiz et al. [8]. The vena contracta factor is $C_{v}=0.82$ (see Sect. 3 and Appendix A). The change in inertance is normalized dividing by the linear contribution $\operatorname{Imag}\left[z_{\text {plate, } \mathrm{L}}\right]$. The non-dimensional corrected resistance and inertance changes due to non-linear effects are:

$$
\begin{aligned}
\Delta R_{\mathrm{NL}} & =2 C_{v}^{2} \Phi \frac{\left(\operatorname{Real}\left[z_{\mathrm{plate}, \mathrm{NL}}\right]-\operatorname{Real}\left[z_{\mathrm{plate}, \mathrm{L}}\right]\right)}{\rho U_{p}}, \\
\Delta I_{\mathrm{NL}} & =\Phi \frac{\left(\operatorname{Imag}\left[z_{\mathrm{plate}, \mathrm{NL}}\right]-\operatorname{Imag}\left[z_{\mathrm{plate}, \mathrm{L}}\right]\right)}{\operatorname{Imag}\left[z_{\text {plate } \mathrm{L}}\right]} .
\end{aligned}
$$

\section{Experiments}

\subsection{Impedance tube setup}

The experimental setup used in this study is an impedance tube with 6 pre-polarized $1 / 4$ inch microphones (type BWSA, sensitivity $50 \mathrm{mV} / \mathrm{Pa}$ ). The tube is made of aluminium with an inner diameter $D_{\mathrm{i}}=50 \mathrm{~mm}$, a wall thickness $t_{\mathrm{w}}=10 \mathrm{~mm}$ and length $l_{\mathrm{t}}=1000 \mathrm{~mm}$. The excitation system is a $25 \mathrm{~W}$ loudspeaker. The six microphones are equally placed at a distance of $175 \mathrm{~mm}$. A relative calibration is performed on the microphones using the microphone closest to the end of the tube (sample side) as the reference microphone. The position of this microphone with respect to the end of the impedance tube is $x_{\text {ref }}=47.7 \mathrm{~mm}$. Details on the setup and the calibration system can be found in the works of Temiz et al. [25] and Kojourimanesh et al. [26]. The micro-slit plates are positioned at the end of the impedance tube through a sample holder. For this study two sample holders are used to compare the effects of three-dimensional effects for a plate confined by the impedance tube from two sides and from one side $\left(L_{\mathrm{h}_{1}}=50 \mathrm{~mm}\right.$ and $\left.L_{\mathrm{h}_{2}}=9 \mathrm{~mm}\right)$. The impedance tube termination with the sample holders is shown in Figure 2. Both the sample holders have a groove for an o-ring to guarantee air-tightness from both the sides of the sample. A script built-in NILabView software controls the signal processing and data acquisition during the measurements. For this study, the sampling rate is $20 \mathrm{kHz}$ for the excitation signal and $10 \mathrm{kHz}$ for recording the input signal. The amplitude of the excitation signal is adjusted automatically until it satisfies the pre-determined pressure value for the reference microphone $\hat{p}\left(x_{\text {ref }}\right)$ within an accuracy of $2 \%$. This amplitude is also used to derive the acoustic velocity at the sample. The calculation of the reflection coefficient at the sample is based on the plane wave assumption. For the evaluation of the reflection coefficient the method from Jang and Hi [27] is used. For each frequency, every microphone records the complex pressure amplitude $\hat{p}(x)$ at position $x$,

$$
\hat{p}(x)=\hat{p}_{+}(x) \exp (-\mathrm{i} k x)+\hat{p}_{-}(x) \exp (\mathrm{i} k x),
$$

with $\hat{p}_{+}$and $\hat{p}_{-}$respectively the amplitudes of the wave travelling in the positive and in the negative directions $x=0$ corresponds to the end of the impedance tube (sample side), $k$ is the complex wavenumber. Taking viscothermal effects into account as proposed in Peters et al. [28], the complex wavenumber is

$$
\begin{aligned}
k= & \frac{\omega}{c_{0}}\left[1+\frac{1-\mathrm{i}}{\sqrt{2} \mathrm{Sh}_{D}}\left(1+\frac{\gamma-1}{\operatorname{Pr}^{0.5}}\right)\right] \\
& -\frac{\omega}{c_{0}}\left[\frac{\mathrm{i}}{\operatorname{Sh}_{\mathrm{D}}^{2}}\left(1+\frac{\gamma-1}{\operatorname{Pr}^{0.5}}-\frac{1}{2} \gamma \frac{\gamma-1}{\operatorname{Pr}}\right)\right],
\end{aligned}
$$

where $\mathrm{i}$ is the imaginary unit, $\mathrm{Pr}$ is the Prandtl number $(\operatorname{Pr}=0.72), \gamma$ is the heat capacity ratio $(\gamma=1.4)$ and $\mathrm{Sh}_{D}$ the Shear number based on the impedance tube diameter $D_{i}$ defined as the ratio of the tube diameter 


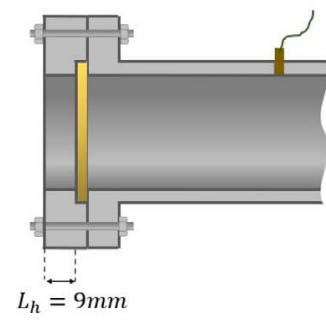

(a)

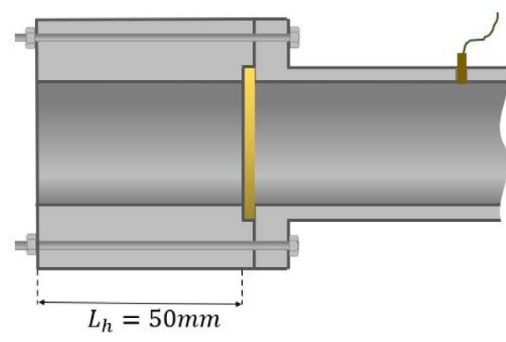

(b)
Figure 2. Impedance tube termination with a) short sample holder of length $L_{\mathrm{h}}=9 \mathrm{~mm}$ and b) long sample holder of length $L_{\mathrm{h}}=50 \mathrm{~mm}$.

and the viscous boundary layer thickness in the tube $\left(\mathrm{Sh}_{d}=D_{i} / \delta_{v}\right)$. The reflection coefficient at the end of the tube $(x=0)$ is [27]

$$
R=\frac{\hat{p}_{-}}{\hat{p}_{+}} .
$$

Experimentally, the closed pipe termination at $x=0$ is used as a reference for the accuracy of the measurements. For an amplitude $\hat{p}\left(x_{\text {ref }}\right)=2 \mathrm{~Pa}$, the maximum deviation from the theoretical value $R=1.000$ is less than $0.3 \%$. The closedend measurements are performed for several excitation amplitudes. It appears that for amplitude $\hat{p}\left(x_{\text {ref }}\right)>23 \mathrm{~Pa}$ the accuracy of the measurements reduces to $1 \%$ up to $f=400 \mathrm{~Hz}$. For $f>400 \mathrm{~Hz}$ this accuracy reduces to around $3 \%$. Hence, the study of the amplitude dependence of the measurements is restricted to $0.4 \mathrm{~Pa}<\hat{p}\left(x_{\text {ref }}\right)<23 \mathrm{~Pa}$. For low frequencies $(f<200 \mathrm{~Hz})$, no significant effects are found increasing the measurement time (number of samples). For $f>700 \mathrm{~Hz}$ the results appear to be less reliable. Therefore, the frequency range of the measurements is restricted to $20 \mathrm{~Hz}<f<700 \mathrm{~Hz}$ corresponding to $1<\mathrm{Sh}<6$.

\subsubsection{Measuring the transfer impedance of the sample and acoustic velocity}

To measure the transfer impedance of the plate the following procedure is followed. At the impedance tube termination an additional pipe segment of the same length of the sample holder is added. The open pipe termination is located at $x_{\text {open }}=x_{0}+L_{\mathrm{h}}+t_{\mathrm{p}}$, with $x_{0}=0$ is the right side of the plate in Figure 2, $L_{\mathrm{h}}$ the length of the holder and $t_{\mathrm{p}}$ the thickness of the sample. The reflection coefficient $R_{\mathrm{o}}$ for the open pipe termination is measured. The dimensionless radiation impedance is calculated using $z_{\mathrm{rad}}=\left(1+R_{\mathrm{o}}\right) /$ $\left(1-R_{\mathrm{o}}\right)$. The tube is then loaded with the sample by the use of a sample holder. The reflection coefficient of the sample-loaded termination $R_{s}$ is measured. The sample-loaded impedance can be calculated in the same way as the radiation impedance as $z_{s}=\left(1+R_{s}\right) /\left(1-R_{s}\right)$. The samples have relatively low porosity. Therefore, the radiation impedance is expected to be much lower than the impedance of the plate. Nevertheless, the radiation effects are taken into account and the dimensionless transfer impedance of the plate $z_{\text {plate }}$ is calculated as the difference between the sample-loaded impedance and the radiation impedance, in formula $z_{\text {plate }}=z_{\mathrm{s}}-z_{\text {rad }}$. The radiation of the room is close to that of a free field. Deviations due to unwanted changes in room acoustics are taken into account repeating the open pipe termination experiments before each set of measurements. One does observe some systematic deviation from free-space radiation as a result of room resonances. Assuming the radiation impedance is in series with the plate impedance, the room effect is corrected for by measuring the radiation impedance. In this open pipe radiation impedance measurement, the sample is replaced by a ring in the sample holder so that the geometry (pipe length, position in the room) is exactly the same as when measuring with a sample. The analysis for non-linear studies is performed for $f \leq 200 \mathrm{~Hz}$. In this analysis of non-linear effects the amplitude $|\hat{u}|$ of the flow velocity is a key parameter. The magnitude of the acoustic velocity at the plate is calculated as $|\hat{u}|=\hat{p}\left(x_{\text {ref }}\right) /\left|z_{\text {plate }}\right|$. Hence, it is assumed that $\hat{p}(x=0) \approx$ $\hat{p}\left(x_{\text {ref }}\right)$. This is certainly accurate at low frequencies $(f<340 \mathrm{~Hz})$ given $x_{\text {ref }} \approx 50 \mathrm{~mm}$.

\subsection{Specifications of the samples}

The acoustical behaviour of short and long slits in micro-slit plates is compared by considering two samples. The geometry of the plates is inspired to the plate in Figure 1. The plates are realized in brass with milling process [29]. Sketches of the plates showing the main parameters are shown in Figure 3. The plates are shown in Figure 4. The external diameter of the plates is $D_{\mathrm{p}}=70 \mathrm{~mm}$, allowing hosting the plates in the holder.

The effective diameter of the portion of the plate where the slits are located is $D_{\mathrm{i}}=50 \mathrm{~mm}$, with $D_{\mathrm{i}}$ the internal diameter of the impedance tube. The total plate thickness is $t_{\mathrm{p}}=5 \mathrm{~mm}$ and the nominal slit width is $b=0.5 \mathrm{~mm}$. The external width of the ditch is $w_{\mathrm{d}, \mathrm{e}}=5 \mathrm{~mm}$, the internal width of the ditch is $w_{\mathrm{d}, \mathrm{i}}=2.25 \mathrm{~mm}$. The ditch thickness is $t_{\mathrm{d}}=2.75 \mathrm{~mm}$. The thickness of the plate at the slit is $t=t_{\mathrm{p}}-t_{\mathrm{d}}=2.25 \mathrm{~mm}$. The slit length is $l_{\mathrm{s}, \text { short }}=15 \mathrm{~mm}$ for short slits and $l_{\mathrm{s} \text {,long }}=35 \mathrm{~mm}$ for long slits. The angle between the internal ditch and the outside ditch is $45^{\circ}$. The plates are realized in such a way that the total length of the slits $P$ is the same. The total slit length is $P=7 \times l_{\text {s, }}$ short $=3 \times l_{\text {s,long }} \approx 105 \mathrm{~mm}$. The porosity (of the portion of the plate in the impedance tube) is $\Phi=2.7 \%$. In order to provide access to the rotary cutter, the ditch length is longer than the slit length, $l_{\mathrm{d}}=l_{\mathrm{s}}+2 \mathrm{~mm}$. The actual widths of the single slits are measured experimentally by means of a digimatic indicator. Maximum deviations from the prescribed dimensions are of the order of $2 \%$ $( \pm 1 \mu \mathrm{m})$ for the plate with short slits and $4 \%( \pm 1 \mu \mathrm{m})$ for the plate with long slits. The measured average of $b$ over the slit length has been used $b=0.505 \mathrm{~mm}$ for short slits and $b=0.520 \mathrm{~mm}$ for long slits. Therefore, the porosity is $\Phi_{\text {short }}=2.7 \%$, for the plate with short slits and and $\Phi_{\text {long }}=2.78 \%$, for the plate with long slits. The edges in contact with the slits are kept as sharp as possible to 

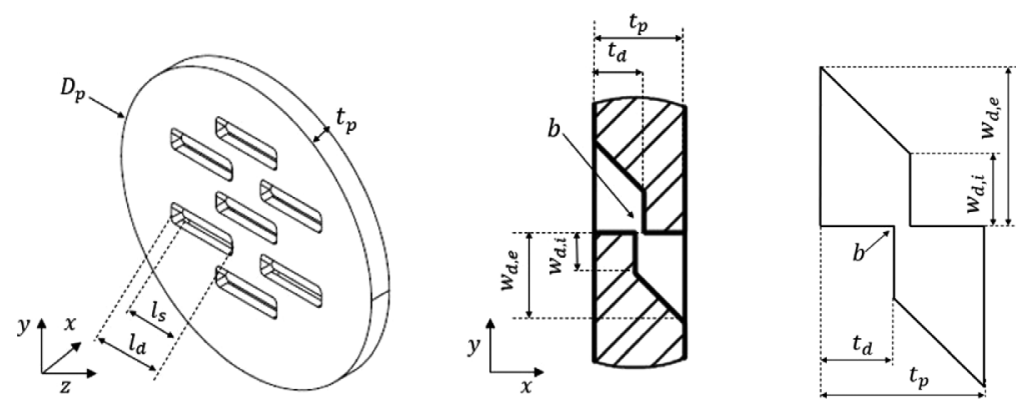

Figure 3. Sketches of the plate and of the cross-section of a single slit with parameters.

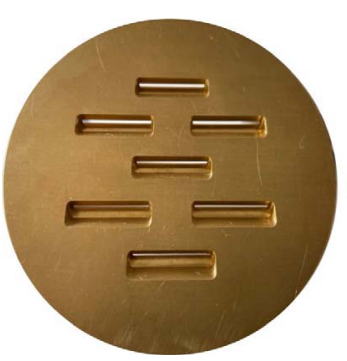

(a)

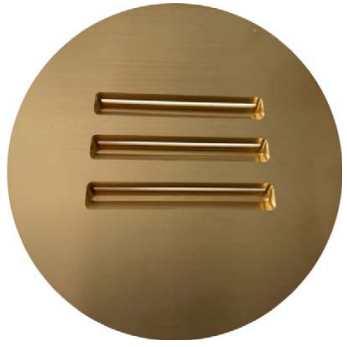

(b)
Figure 4. Picture of the samples: a) plate with short slits and b) plate with long slits.

remove effects due to the rounding of the edges. Observations under microscope (magnification $50 \times$ ) did not show any significant deviation from sharp edges.

\section{Numerical model}

In this section, a two-dimensional numerical model is proposed. Solution of the linearized Navier-Stokes equations for an incompressible flow is considered. Consider the cross-section of the plates shown in Figure 3. A single slit of width $b$ in a confinement channel of width $a$ is considered. The confinement channel width is chosen such as the porosity of the two-dimensional model $\Phi_{2 \mathrm{D}}$ is equal to the actual porosity of the plate $\Phi$. The plate of thickness $t_{\mathrm{p}}$ is enclosed between an upstream and a downstream channel of lengths $L_{\mathrm{u}}$ and $L_{\mathrm{d}}$. The computational domain is shown in Figure 5. The domain is divided into three sub-domains for meshing purposes. Continuity of pressure and velocity is assumed at the boundaries between the calculation domains. All the lengths are made dimensionless with the slit width $b$. The dimensionless plate thickness is $t_{p}^{*}=t_{p} /$ $b=5 \mathrm{~mm} / 0.5 \mathrm{~mm}=10$ (Distance BC in Fig. 5). The confinement channel width is $a^{*}=a / b=18 \mathrm{~mm} /$ $0.5 \mathrm{~mm}=36$ (Segment AH in Fig. 5). The lengths of the channels upstream (Segment AB in Fig. 5) and downstream the channel (Segment CD in Fig. 5) are chosen to have $L_{u}^{*}=L_{d}^{*}=2 a^{*}$. The upstream face of the plate is located at $x_{\mathrm{BG}}^{*}=-4.5$ and the slit opening at $x^{*}=0$. The downstream face of the plate is located at $x_{\mathrm{CF}}^{*}=5.5$ and the end of the slit at $x^{*}=1$. The upstream side of domain $\mathrm{AH}$ is located at $x_{\mathrm{AH}}^{*}=-L_{\mathrm{u}}^{*}-x_{\mathrm{BG}}^{*}=-72-4.5=-76.5$.
The downstream side of domain $\mathrm{DE}$ is located at $x_{\mathrm{DE}}^{*}=$ $x_{\mathrm{CF}}^{*}+L_{d}^{*}=4.5+1+72=77.5$. Therefore, the domain extends between $x^{*}=x / b=\left[x_{\mathrm{AH}}^{*}, x_{\mathrm{DE}}^{*}\right]=[-76.5,77.5]$ and $y^{*}=y / b=[0,36]$.

The low He number approximation is made. The incompressible Linear Navier-Stokes equations for a 2D domain in a dimensionless form in the frequency domain are hereby presented for a radial frequency $\omega$ :

$$
\frac{\partial \hat{u}^{*}}{\partial x^{*}}+\frac{\partial \hat{v}^{*}}{\partial y^{*}}=0,
$$

$$
\begin{aligned}
& i \hat{u}^{*}=-\frac{\partial \hat{p}^{*}}{\partial x^{*}}+\frac{1}{2 \operatorname{Sh}_{b}^{2}}\left(\frac{\partial^{2} \hat{u}^{*}}{\partial x^{* 2}}+\frac{\partial^{2} \hat{u}^{*}}{\partial y^{* 2}}\right), \\
& i \hat{v}^{*}=-\frac{\partial \hat{p}^{*}}{\partial y^{*}}+\frac{1}{2 \operatorname{Sh}_{b}^{2}}\left(\frac{\partial^{2} \hat{v}^{*}}{\partial x^{* 2}}+\frac{\partial^{2} \hat{v}^{*}}{\partial y^{* 2}}\right),
\end{aligned}
$$

with $x^{*}=x / b$ and $y^{*}=y / b$. The dimensionless velocity $\left(\hat{u}^{*}, \hat{v}^{*}\right)$ is $(\hat{u} / b \omega, \hat{v} / b \omega)$ and the dimensionless pressure is $\hat{p}^{*}=\hat{p} /\left(\rho(b \omega)^{2}\right)$. These equations are implemented in COMSOL Multiphysics as user defined equations (PDE) and solved. For the inlet segment $\mathrm{AH}$ and outlet segment $\mathrm{DE}$ uniform pressures are imposed, $p_{\mathrm{AH}}^{*}=1$ and $p_{\mathrm{DE}}^{*}=0$. At the segments BC and FG (walls) no-slip boundary conditions are applied, i.e. $\left(u^{*}, v^{*}\right)=(0,0)$. At the segments $\mathrm{AB}, \mathrm{CD}, \mathrm{FE}, \mathrm{GH}$ slip boundary conditions are implemented to simulate the hydro-dynamical interaction between neighbouring slits in micro-slit plate with multiple slits. In the present case, the slits are very thin $(b / a \ll 1)$ therefore a slip boundary condition is used instead of a periodic boundary condition. A slip condition implies an equality of the velocities at the corresponding boundaries. Due to the dimensions of the slits, the deviation from a symmetric case is small. An unstructured mesh of quadratic triangular elements is used, with a finer mesh at the walls with no-slip conditions. The mesh inside the plate domain (Domain 2) is finer with the maximum element size is $M_{\mathrm{el}} / b=5 \times 10^{-2}$ and the minimum is $m_{\mathrm{el}} / b=1 \times 10^{-4}$. A mesh convergence study shows a quadratic convergence of the results using the average velocity in a cross-section (line) calculated at a location $x^{*}=-1.7 a^{*}$. Far from the slit, the acoustic pressure is uniform in the cross-section and the amplitude of the pressure depends linearly on the position along the duct 


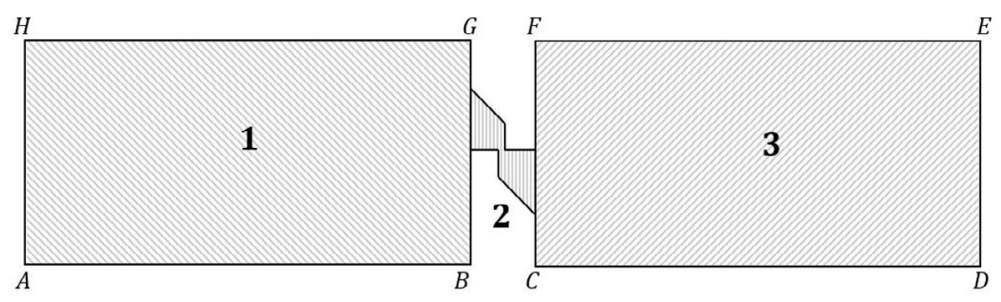

Figure 5. Two-dimensional computational domain for the plate of thickness $t_{\mathrm{p}}$ and slit width $b$ enclosed between an upstream and a downstream channel. Three sub-domains (1-2-3) are defined for meshing purposes.

(parallel flow behaviour described in Aulitto et al. [20]). For $-1.7 a^{*}<x^{*}<-1.1 a^{*}$, one has $\hat{p}^{*}\left(x^{*}\right)=\hat{\alpha} x^{*}+\hat{\beta}$. For $1.1 \mathrm{a}^{*}<x^{*}<1.7 \mathrm{a}^{*}$, one has $\hat{p}^{*}\left(x^{*}\right)=\hat{\gamma} x^{*}+\hat{\delta}$. The complex constants can be determined by a linear fit of the pressure data obtained by numerical simulations for these regions far from the discontinuity. For a $\mathrm{Sh}_{b}=2.5$ (corresponding to $f=120 \mathrm{~Hz}$ for a slit width $b=0.5 \mathrm{~mm}$ ), the linear fit gives a coefficient of determination $1-R^{2}=10^{-6}[30]$. The transfer impedance of the slit $Z_{\text {slit }}^{*}$ is determined by $Z_{\text {slit }}^{*}=\frac{\hat{\beta}-\hat{\delta}}{\hat{U}^{*}}$ with $\hat{U}^{*}$ being the flux calculated in a generic section of the channel upstream the slit, defined as $\hat{U}^{*}=w^{*}<\hat{u}^{*}>b^{*}$, with $w^{*}=1$. From $Z_{\text {slit }}^{*}$ one can derive the dimensionless transfer impedance of the slit $z_{\text {slit }}$ as

$$
z_{\text {slit }}=\frac{2 v \mathrm{Sh}_{b}^{2}}{b c} Z_{\text {slit }}^{*}
$$

with $\mathrm{Sh}_{b}$ the Shear number based on the slit width. Tests for several channel widths $a$ are performed. It appears that the resistance of the slit is independent of the porosity of the plate. Reducing of a factor 7 the porosity, the resistance increases by less than $0.7 \%$. The inertia of the plate changes with changing the porosity. For a drastic reduction of the porosity, by a factor 7 , the inertia increases by $30 \%$. For small changes of the porosity around the nominal value, as the difference found between the two samples, the change is negligible. One can conclude that the transfer impedance of the slit appears to be only weakly dependent on the porosity $\Phi$.

\section{Results}

\subsection{Results in linear regime}

In Figure 6, the resistive and the reactive part of the impedance are shown for long and short slits in the range $2.5<\mathrm{Sh}_{b}<6$ corresponding to $120 \mathrm{~Hz}<f<700 \mathrm{~Hz}$. The slit impedance of a single slit is displayed, $z_{\text {slit }}=z_{\text {plate }} \Phi$, calculated using $\Phi_{\text {short }}$ and $\Phi_{\text {long }}$ for the short slits and long slits, respectively. The amplitude of the acoustic waves is $\hat{p}\left(x_{\text {ref }}\right)=2 \mathrm{~Pa}$. Frequencies below $120 \mathrm{~Hz}$ are excluded because of the presence of non-linear effects that will be discussed in Section 5.2. Frequencies above $700 \mathrm{~Hz}$ are ignored due to uncertainties in the measurements. The results are presented for a sample holder $L_{h}=9 \mathrm{~mm}$.

It appears that the maximum deviation between the resistance of long and short slits is of the order of $4 \%$. This deviation is most probably due to the difference in slit width between the two plates and could be due to differences in edge sharpness. At low $\mathrm{Sh}_{b}$ the resistance scales with $1 / b^{2}$. An uncertainty of $2 \%$ in $b$ explains a difference of the order of $4 \%$. The influence of the length of the slits is negligible. The difference between the inertance of short and long slits is of the order of $10 \%$. The inertance of short slits is lower than the inertance of long slits. This is due to three-dimensional effects due to the geometry of the slits. Three-dimensional effects do not change significantly for the plate confined from both sides (holder with $L_{\mathrm{h}}=50 \mathrm{~mm}$ ) or for a free plate (holder with $L_{\mathrm{h}}=9 \mathrm{~mm}$ ). The effect of the sample holder length is negligible for $f<500 \mathrm{~Hz}$. At higher frequencies the data with the long holder show oscillating frequency dependency. This is to expected to be connected to the presence of a table in the measurement room. Therefore, the small holder is chosen to display the results. In Figure 6 , the two-dimensional numerical model for a single slit is compared to the experimental results. It appears that the 2D numerical model of a single slit predicts (within few percentage of accuracy) the impedance (both resistive and reactive part) of long slits and the resistance of short slits. In the same figure, the semi-analytical model for high Shear numbers of Aulitto et al. [20] for a plate with square sharp edges with thickness $t_{\mathrm{p}}=0.5 \mathrm{~mm}$ is shown. For the semianalytical model, the plate thickness is assumed to be the same as the slit width. It appears that the model predicts reasonably well the resistance of the plate with the geometry proposed in this work. This confirms that the resistance of micro-slit plate is a local effect, strongly affected by the geometry of the edge and less sensitive to the global geometry of the plates [20]. The inertance obtained when assuming $b=t_{\mathrm{p}}=0.5 \mathrm{~mm}$, on the other hand, is much lower than that of the plates used in this study. In conclusion, in the linear regime $\left(\mathrm{Sh}_{\mathrm{b}}>2.5\right)$, the difference between the resistance (or resistive part of the impedance) for short and long slits is small. The inertance (or reactive part) of short slits is smaller (within 10\%) than long slits due to three-dimensional effects. Experiments with two sample holders exclude a dependence on the confinement of the plate. It appears that the two-dimensional numerical model well predicts the resistance of long and short slits.

\subsection{Results in non-linear regime}

For Shear numbers $\left(\mathrm{Sh}_{\mathrm{b}}<2.5\right)$ deviations from the numerical model for both short and long slits appear. These deviations depend on the amplitude of the acoustic waves. 


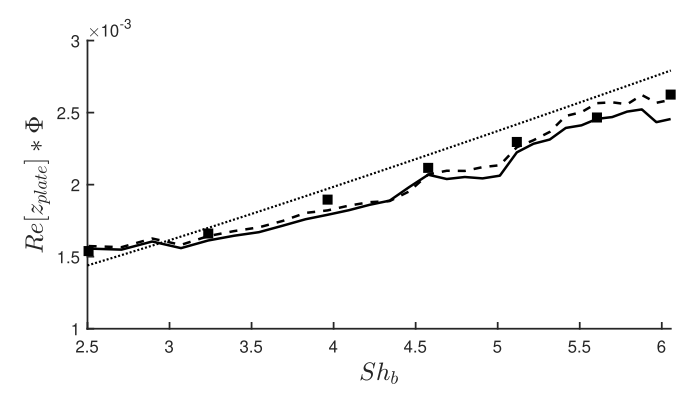

(a)

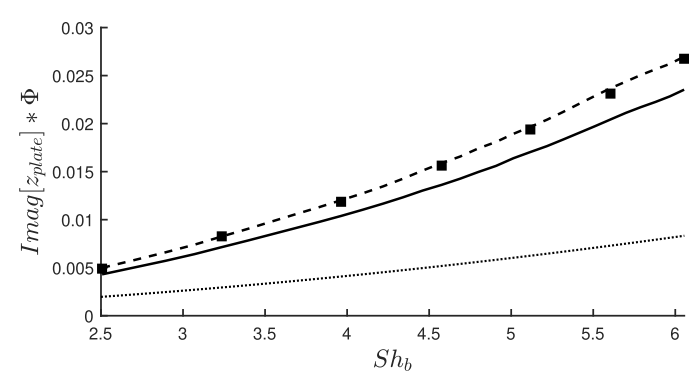

(b)

Figure 6. Comparison of the a) slit resistance and b) slit inertance for short (-) and long (- - -) slits as a function of Shear number in linear regime. The numerical model $(\boldsymbol{\square})$ and the semi-analytical model for a plate with square sharp edges (. . . .) are shown [20].

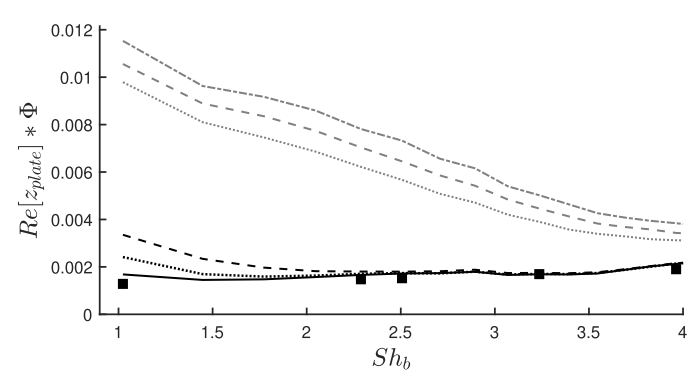

(a)

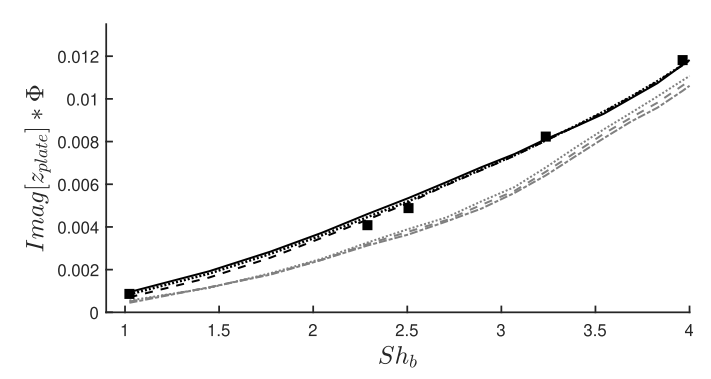

(b)

Figure 7. Comparison of the a) slit resistance and b) slit inertance for long slits compared to 2D model ( $\boldsymbol{\square}$ ) as function of Shear number for several amplitudes at the reference microphone. Low amplitudes: (-) $0.4 \mathrm{~Pa},(. \ldots$.) 1 Pa, (- - -) 2 Pa. High amplitudes: (-) $17 \mathrm{~Pa},(. .) .20 \mathrm{~Pa},(--$-)23 Pa.

This is shown for long slits for several amplitudes in Figure 7. These deviations at low frequencies are due to non-linear effects and decrease for increasing Shear numbers. At $\mathrm{Sh}_{\mathrm{b}}<2.5$ for $\hat{p}\left(x_{\text {ref }}\right)=2 \mathrm{~Pa}$ one has $\mathrm{St}_{\mathrm{b}}<0.4$. Non-linear effects impact both the inertance and the resistance of the slit. In particular, at the lowest frequency, the resistance for the highest amplitude $\left(\hat{p}\left(x_{\text {ref }}\right)=23 \mathrm{~Pa}\right)$ is 7 times higher than the resistance at the lowest amplitude $\left(\hat{p}\left(x_{\mathrm{ref}}\right)=0.4 \mathrm{~Pa}\right)$. The inertance is reduced by almost a factor 2, as expected from the model of Ingard and Ising [21].

In this study, sound generation as higher harmonics due to non-linearity is not considered. For long slits, the plots of $\Delta \mathrm{R}_{\mathrm{NL}}$ and $\Delta \mathrm{I}_{\mathrm{NL}}$ are provided as function of the Strouhal number for several amplitudes of the acoustic waves. On the lower horizontal scale, the Strouhal number based on the slit width $\mathrm{St}_{b}$ is used. On the upper horizontal scale, the Strouhal number based on the plate thickness at the slit $\mathrm{St}_{t}$ appears. In Figure 8, results are shown for high amplitudes of the acoustic waves $\left(\hat{p}\left(x_{\text {ref }}\right) \geq 10 \mathrm{~Pa}\right)$. Both the change in resistance and inertance due to non-linearity increase (in absolute value), as expected, for decreasing Strouhal number $\left(\mathrm{St}_{b}=\omega b / U_{p}\right)$. For $1 / \mathrm{St}_{b} \gg 1$ the change in resistance $\Delta \mathrm{R}_{\mathrm{NL}}$ is approaching the theoretical quasisteady potential flow limit with correction for the viscous boundary layer for $\mathrm{St}_{b} \rightarrow 0$. The change in inertance approaches the value $\Delta \mathrm{I}_{\mathrm{NL}}=-0.5$. The correction in the inertance due to non-linear effects is almost half the inertance in the linear case. Figure 9 compares the non-linear resistance and inertance for long slits to that of short slits for high amplitudes $\left(\hat{p}\left(x_{\text {ref }}\right) \geq 10 \mathrm{~Pa}\right)$. In Figure 10, the results are shown for low and moderate amplitudes for short and long slits $\left(\hat{p}\left(x_{\text {ref }}\right) \leq 6 \mathrm{~Pa}\right)$. These results display some Shear number dependency, which is less pronounced at higher amplitudes.

It can be seen that the resistance changes due to nonlinearity are almost identical for long and short slits, both for high and moderate amplitudes. Non-linear effects on the inertance of short slits are, in absolute value, smaller than for long slits. At moderate amplitudes $\left(1 / \mathrm{St}_{b}<20\right)$ one observes a weak non-linear behaviour reported by Ingard and Labate [19]. The vortices are formed at the edges, but they remain close to the slit. For $1 / \mathrm{St}_{b}>20$ and for higher amplitudes, the vortices start moving away from the slits. One observes in this region differences between long and short slits. For very high amplitudes $1 / \mathrm{St}_{b}>50$ the behaviour of the inertia of short and long slits is completely different. The flow for the short slits becomes essentially three-dimensional while it remains approximately two-dimensional for the long slits because of the confinement in the impedance pipe and hydrodynamical interactions between slits. Differences for high amplitudes are most probably due to different behaviour of the synthetic jet (zero net mass flow) in 3D depending on the length of the aperture [31]. Difference in the slits (such as small surface perturbations in the edges) can generate different behaviour of the jet and different spatial 


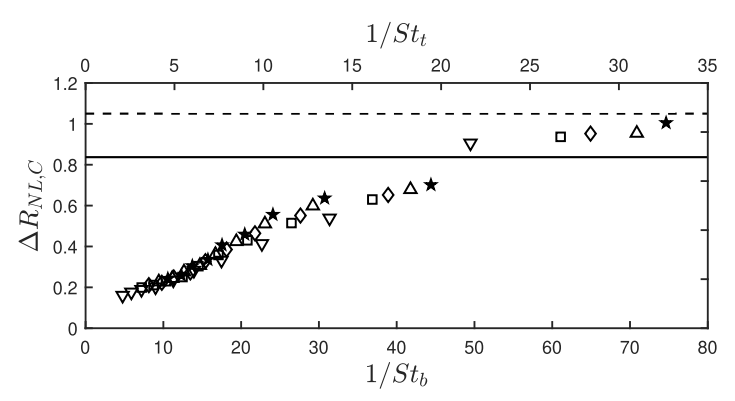

(a)

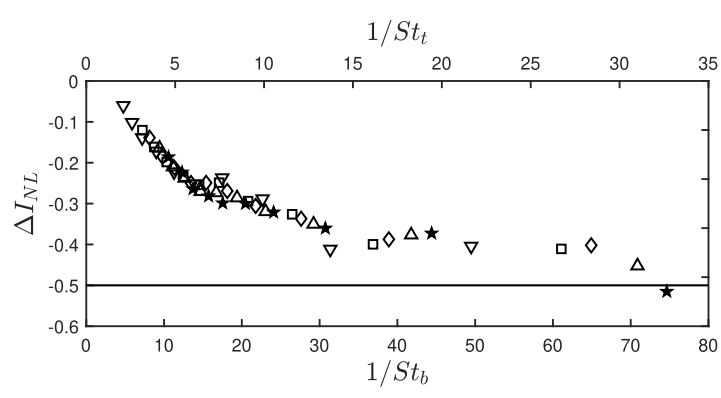

(b)

Figure 8. Change in a) slit resistance and b) slit inertance due to non-linearity for long slits as function of $\mathrm{St}_{b}$ and $\mathrm{St}_{t} \mathrm{for}_{\mathrm{several}}$ amplitudes at the reference microphone: $10 \mathrm{~Pa}(\triangle), 15 \mathrm{~Pa}(\square), 17 \mathrm{~Pa}(\diamond), 20 \mathrm{~Pa}(\triangle), 23 \mathrm{~Pa}(\star)$ ). In a) quasi-steady potential flow theory $(-)$ and quasi-steady potential flow theory corrected for the effect of quasi-steady viscous boundary layer (- - - -); in b) Limit proposed by Ingard and Ising [21] (-).

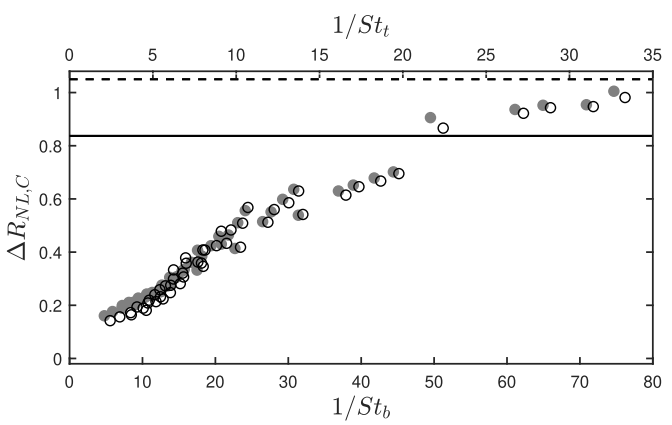

(a)

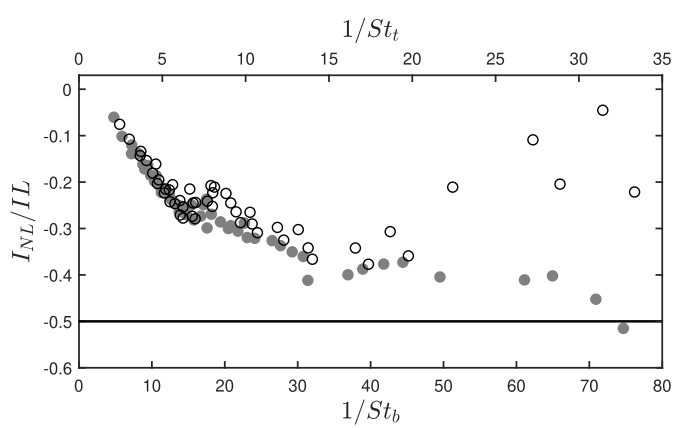

(b)

Figure 9. Change in a) slit resistance and b) slit inertance due to non-linearity for long $(\bigcirc)$ and short $(\bigcirc)$ slits as function of $\mathrm{St}_{b}$ and $\mathrm{St}_{t}$ for several amplitudes at the reference microphone $\left(\hat{p}\left(x_{\mathrm{ref}}\right) \geq 10 \mathrm{~Pa}\right)$. Lines are defined as in Figure 8 .

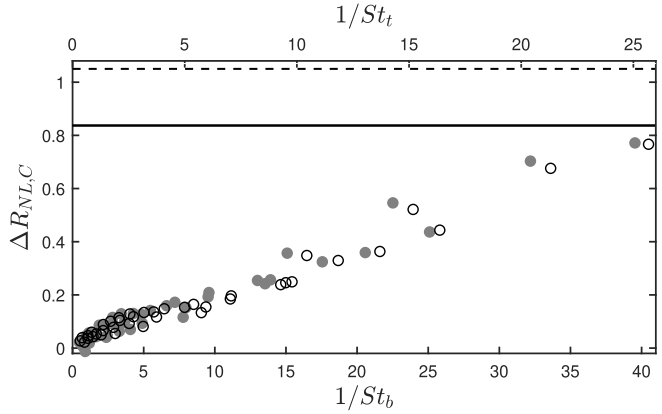

(a)

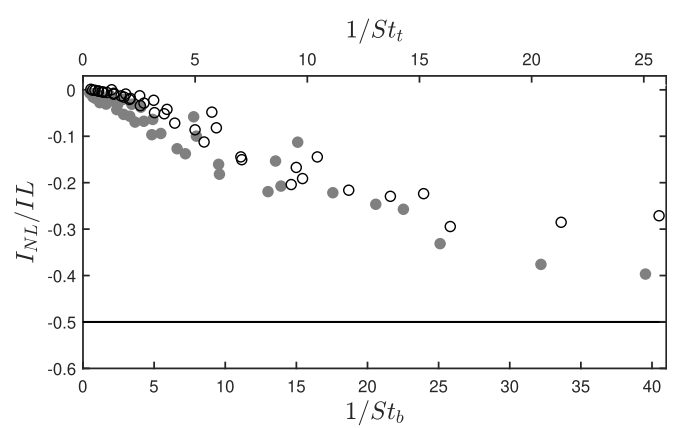

(b)

Figure 10. Change in a) slit resistance and b) slit inertance due to non-linearity for long ( () and short $(\bigcirc)$ slits as function of $\mathrm{St}_{b}$ and $\mathrm{St}_{t}$ for several amplitudes at the reference microphone $\left(\hat{p}\left(x_{\mathrm{ref}}\right) \geq 6 \mathrm{~Pa}\right)$. Lines are defined as in Figure 8 .

evolution of three-dimensional vortices. Examples of complex three-dimensional behaviour of jet formed by a slit are the axis switching and forking $[32,33]$. The behaviour of short and long slits for low $\mathrm{St}_{b}$ is different. In axis switching, the lateral ends of the jet will curve towards the symmetry axis, so that, within a distance comparable to the jet width, a almost plane jet will be formed in a direction normal to the original jet. In forking, the planar jet breaks down into separate jets. These observations confirm that while the resistance is determined locally, the inertance is a more global flow effect.

\section{Discussion and conclusions}

In this work, the effect of slit length on the linear and the non-linear acoustic transfer impedance of two accurately manufactured micro-slit plates has been investigated. 
The study is limited to sharp edges. Experimental data are obtained by impedance tube measurements. The frequency range of interest is $20 \mathrm{~Hz}<f<700 \mathrm{~Hz}$ corresponding to Shear numbers in the range $1<\mathrm{Sh}_{b}<6$. As for circular micro-perforations, the dissipation of sound occurs mainly at the edges of the micro-slits [20,25]. Both in the linear and non-linear regime, the resistance of a single slit is independent of the porosity of the plate and on the slit length. Therefore, by dividing the transfer impedance of a slit by the porosity of the plate, one obtains an accurate prediction of the plate resistance for micro-slit plates. In the linear regime, an accurate prediction of the resistance of the plate is obtained using a two-dimensional numerical solution of the linearized Navier-Stokes equations in a single slit. In the non-linear regime, the plate resistance is strongly dependent on the amplitude of the acoustic waves. A simple quasi-steady model provides an order of magnitude for the asymptotic value of the non-linear resistance. Combining the results obtained in the present work for a specific geometry with the earlier studies on linear acoustical properties of micro-slits (see Aulitto et al. [20] and Temiz et al. [25]) one can conclude that the independence of resistance on the slit length is due to the fact that the resistance is mainly determined by the local acoustic flow around the edges. Therefore, this observation is independent of the exact slit geometry. The inertance of slits is sensitive to the acoustic flow outside the perforations. This explains the strong difference between the behaviour of slits and circular micro-perforations [20]. Also, one observes a small but yet significant reduction of the inertance of short slits with respect to long slits. The linear inertance of long slits is accurately predicted by the locally two-dimensional incompressible numerical model. For moderate amplitudes, the non-linear inertance is not strongly affected by the slit length. For high amplitudes, one observes a strong deviation between the inertance of short and long slits. For long slits, the inertance at very high amplitudes is reduced by a factor two with respect to the linear case, as predicted by the intuitive model of Ingard and Ising [21]. For short slits, the amplitude dependency of the inertance is more complex due to three-dimensional effects. The weak dependency of the inertia on the slit length is also expected to be independent of the exact geometry of the slits. The non-linear behaviour depends on the vena contracta factor, which is dependent on the details of the slit geometry $[8,34]$. However, the fact that the slit length influences more the nonlinear effects on the inertia than the resistance is expected to be independent of details of the slit geometry. Hence, most conclusions drawn from the present study are expected to be quite general.

\section{Acknowledgments}

The authors wish to thank V. Saxena and M. Kojourimanesh for the help with the experimental setup and the preliminary measurements.

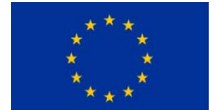

This work is part of the Marie SkłodowskaCurie Initial Training Network Pollution Know-How and Abatement (POLKA). We gratefully acknowledge the financial support from the European Commission under call H2020-MSCA-ITN-2018 (project number: 813367).

\section{Conflict of interest}

The authors declare no conflict of interest.

\section{References}

1. D.-Y. Maa: Theory of microslit absorbers. Acta Acustica 25, 6 (2000) 481-485.

2. H. Ruiz, C.C. Claeys, E. Deckers, W. Desmet: Numerical and experimental study of the effect of microslits on the normal absorption of structural metamaterials. Mechanical Systems and Signal Processing 70 (2016) 904-918.

3. T.G. Zieliński, F. Chevillotte, E. Deckers: Sound absorption of plates with micro-slits backed with air cavities: Analytical estimations, numerical calculations and experimental validations. Applied Acoustics 146 (2019) 261-279.

4. Sontech AB. Acustimet plates ${ }^{\circledR}$. Kungsängen, Sweden. https://www.sontech.se/product-page/acustimet.

5. S. Allam, M. Åbom: A new type of muffler based on microperforated tubes. Journal of vibration and acoustics 133, 3 (2011) 031005.

6. F. Auriemma: Acoustic performance of micro-grooved elements. Applied Acoustics 122 (2017) 128-137.

7.M.A.A. Dah-You: Microperforated panel at high sound intensity. Acta Acustica 1 (1996).

8. M.A. Temiz, J. Tournadre, I.L. Arteaga, A. Hirschberg: Nonlinear acoustic transfer impedance of micro-perforated plates with circular orifices. Journal of Sound and Vibration 366 (2016) 418-428.

9. P. Cobo, C. de la Colina, F. Simon: On the modelling of microslit panel absorbers. Applied Acoustics 159 (2020).

10. F. Auriemma: Study of a new highly absorptive acoustic element. Acoustics Australia 45, 2 (2017) 411-419.

11. U.R. Kristiansen, T.E. Vigran: On the design of resonant absorbers using a slotted plate. Applied Acoustics 43, 1 (1994) 39-48.

12.R.T. Randeberg: Adjustable slitted panel absorber. Acta Acustica united with Acustica 88, 4 (2002) 507-512.

13. X. Dai, X. Jing, X. Sun: Vortex shedding and its nonlinear acoustic effect occurring at a slit. AIAA Journal 49, 12 (2011) 2684-2694.

14. X. Jing, X. Sun: Sound-excited flow and acoustic nonlinearity at an orifice. Physics of Fluids 14, 1 (2002) 268-276.

15. J. Su, J. Rupp, A. Garmory, J.F. Carrotte: Measurements and computational fluid dynamics predictions of the acoustic impedance of orifices. Journal of Sound and Vibration 352 (2015) 174-191.

16. Z. Chen, Z. Ji, H. Huang: Acoustic impedance of perforated plates in the presence of bias flow. Journal of Sound and Vibration 446 (2019) 159-175.

17. A. Komkin, A. Bykov, M. Mironov: Experimental study of nonlinear acoustic impedance of circular orifices. The Journal of the Acoustical Society of America 148, 3 (2020) 13911403 . 
18. A. Cummings, W. Eversman: High amplitude acoustic transmission through duct terminations: Theory. Journal of Sound and Vibration 914 (1983) 503-518.

19. U. Ingård, S. Labate: Acoustic circulation effects and the nonlinear impedance of orifices. The Journal of the Acoustical Society of America 22, 2 (1950) 211-218.

20. A. Aulitto, A. Hirschberg, I. Lopez Arteaga: Influence of geometry on acoustic end-corrections of slits in microslit absorbers. The Journal of the Acoustical Society of America 149, 5 (2021) 3073-3085.

21. U. Ingard, H. Ising: Acoustic nonlinearity of an orifice. The journal of the Acoustical Society of America 42, 1 (1967) 617.

22. J.H. Spurk, N. Aksel: Introduction into theory of flows. 8. rev. and enl. ed.; Stroemungslehre. Einfuehrung in die Theorie der Stroemungen, 2010.

23. D. Gilbarg: Jets and cavities. Fluid Dynamics/Strömungsmechanik, Springer. 1960, pp. 311-445.

24. P.M. Morse, K.U. Ingard: Theoretical acoustics, Princeton University Press. 1986

25. M.A. Temiz, I. Lopez Arteaga, G. Efraimsson, M. Åbom, A. Hirschberg: The influence of edge geometry on end-correction coefficients in micro perforated plates. The Journal of the Acoustical Society of America 138, 6 (2015) 3668-3677.

26. M. Kojourimanesh, V. Kornilov, I.L. Arteaga, P. de Goey: Thermo-acoustic flame instability criteria based on upstream reflection coefficients, Combustion and Flame 225 (2021) $435-443$.

27. S.H. Jang, J.G. Ih: On the multiple microphone method for measuring in-duct acoustic properties in the presence of mean flow. The Journal of the Acoustical Society of America 103, 3 (1998) 1520-1526.

28. M.C.A.M. Peters, A. Hirschberg, A.J. Reijnen, A.P.J. Wijnands: Damping and reflection coefficient measurements for an open pipe at low mach and low Helmholtz numbers. Journal of Fluid Mechanics 256 (1993) 499-534.

29. C. Suryanarayana: Mechanical alloying and milling. Progress in Materials Science 46, 1-2 (2001) 1-184.

30. S.A. Glantz, B.K. Slinker: Primer of Applied Regression \& Analysis of Variance, ed. McGraw-Hill Inc, New York. 2001.

31. M. Amitay, F. Cannelle: Evolution of finite span synthetic jets. Physics of Fluids 18, 5 (2006) 054101.

32. E.J. Gutmark, F.F. Grinstein: Flow control with noncircular jets. Annual Review of Fluid Mechanics 31, 1 (1999) 239-272.

33. W.C. Reynolds, D.E. Parekh, P.J.D. Juvet, M.J.D. Lee: Bifurcating and blooming jets. Annual review of fluid mechanics 35, 1 (2003) 295-315.

34. E.M.T. Moers, D. Tonon, A. Hirschberg: Strouhal number dependency of the aero-acoustic response of wall perforations under combined grazing-bias flow. Journal of Sound and Vibration 389 (2017) 292-308.

35. B. Thwaites: On two solutions of the boundary-layer equations. In: 50 Jahre Grenzschichtforschung, Wiesbaden: Vieweg+Teubner Verlag. 1955, pp. 210-215.

\section{Appendix A}

\section{A.1 Vena contracta factor for potential flow}

At high amplitudes the flow detaches at the edges and then forms a free jet that contracts. The computation of jet flow is difficult, but in plane potential flow the problem of free jet can be solved by conformal mapping. The contraction factor of the jet (vena contracta factor) can be

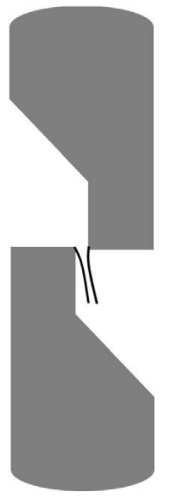

(a)

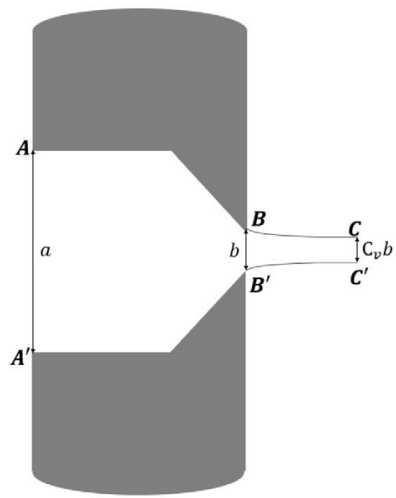

(b)
Figure A.1. Schematic representation of the slit and zoomed simplified model for the contraction of the free jet after the plate.

calculated for several geometries following approaches found in literature $[22,23]$. In Figure A.1 the geometry of a single slit is compared with a simplified model that will be used to calculate the vena contracta factor using the method of Spurk and Aksel [22]. As defined in Section 4, the porosity of the two-dimensional model is $\Phi_{2 \mathrm{D}}=b / a$. The simplified geometry is connected to the idea that, for low $b / a$, the main parameter is the $45^{\circ}$ angle between the two sides of the slit, because the interaction of the jet with the walls can be neglected.

The emerging jet contracts from the cross-section $B-B^{\prime}$ of width $b$ to the cross-section $C-C^{\prime}$ of width $C_{v} b$, with $C_{v}$ the vena contracta factor. At section $C-C^{\prime}$ the pressure inside the jet is equal to the ambient pressure, since the curvature of the streamlines vanishes. From the Bernoulli's equation, the velocity on the boundary of the jet is

$$
u_{0}=\sqrt{\frac{2}{\rho}\left(p_{1}-p_{0}\right)},
$$

where $p_{1}$ and $p_{0}$ are the pressure before the slit and after the slit, respectively. The duct can be associated to a region in the complex $z$-plane by $z=x+\mathrm{i} y$, with $\mathrm{i}^{2}=-1$ and spatial coordinates $(x, y)$. In order to determine the shape of the free jet, a mapping resulting from the definition of the complex conjugate velocity can be used

$$
\zeta=f(z)=\frac{\mathrm{d} F}{\mathrm{~d} z}=u-\mathrm{i} v=w .
$$

Using conformal mapping, the flow region in the duct can be mapped into a velocity plane, the so-called hodograph plate. For small porosity $(b / a \ll 1)$ the flow can be generated from the superposition of a source of strength $4 C_{v} b u_{0}$ at $w=(u-\mathrm{i} v)=0$ and sinks of $2 C_{v} b u_{0}$ at $w=-1,+1$, $-\mathrm{i},+\mathrm{i}$, with $u_{0}$ the velocity at the edge of the jet. The complex potential is

$$
\begin{aligned}
F=\frac{C_{v} b u_{0}}{\pi}[2 \ln (w)- & \ln \left(w+u_{0}\right)-\ln \left(w-u_{0}\right)-\ln \left(w+\mathrm{i} u_{0}\right) \\
- & \left.\ln \left(w-\mathrm{i} u_{0}\right)\right] .
\end{aligned}
$$


In the present work the limit for $b / a \ll 1$ is considered. The mapping function $z=z(\zeta)$ has to be calculated to determine the free surface in the $z$-plane. From equation (A.2) follows that

$$
z=\int \frac{\mathrm{d} F}{\zeta}=\int \frac{\mathrm{d} F}{\mathrm{~d} \zeta} \frac{\mathrm{d} \zeta}{\zeta} .
$$

The solution to this integral is

$$
\begin{aligned}
z= & \frac{C_{v} b u_{0}}{\pi}\left[-\frac{2}{w}+\frac{1}{u_{0}} \ln \left(\frac{w+u_{0}}{w-u_{0}}\right)-\frac{i}{u_{0}} \ln \left(\frac{w+\mathrm{i} u_{0}}{w-\mathrm{i} u_{0}}\right)\right] \\
& + \text { constant. }
\end{aligned}
$$

The integration constant can be found assuming $w=$ $(1+i) u_{0} / \sqrt{2}$ at $z=0+\mathrm{i} b$ and $w=u_{0}$ at $z=\infty+\mathrm{i} C_{v} b / 2$. It follows that the vena contracta factor in the limit $b / a \ll 1$ is $C_{v}=0.82$.

\section{Appendix B}

\section{B.1 Quasi-steady incompressible flow}

Assuming a quasi-steady incompressible flow with a free jet of vena contracta factor $C_{v}$, the pressure difference $\Delta p(t)$ across the plate resulting from the oscillating flow velocity $u_{p}(t)=U_{p} \cos (\omega t)$ is:

$$
\Delta p(t)=\frac{1}{2} \rho u_{j}\left|u_{j}\right|
$$

where $\rho$ is the air density and $u_{j}$ is the free jet velocity. For the continuity of the velocity one has that $\hat{u}_{j}=u_{p} / C_{v}$ and $u_{p}=u / \Phi$ where $u_{p}(t)=U_{p} \cos (\omega t)$ is the cross-sectional averaged acoustical velocity in the perforation, $u(t)=U \cos (\omega t)$ is the cross-sectional averaged acoustical velocity in the pipe upstream the plate and $\Phi$ is the porosity. Hence, the pressure difference across the plate is

$$
\Delta p=\frac{1}{2} \rho u_{j}\left|u_{j}\right|=\frac{1}{2} \rho \frac{u_{p}\left|u_{p}\right|}{C_{v}^{2}}=\frac{1}{2} \rho \frac{u|u|}{C_{v}^{2} \Phi^{2}} .
$$

The instantaneous power dissipated $P_{w}$ is given by

$$
P_{w}=\Delta p u A_{i},
$$

where $A_{i}=\pi\left(\frac{D_{i}}{2}\right)^{2}$ is the pipe cross-section. Assuming a harmonically oscillating velocity in the pipe $u_{p}$, one finds for the time-averaged dissipated power

$$
\bar{P}_{w}=A_{i} \frac{4}{T} \int_{0}^{T / 4} \Delta p u \mathrm{~d} t=A_{i} \frac{\rho \Phi U_{p}^{3}}{2 C_{v}^{2}} \frac{4}{T} \int_{0}^{T / 4} \cos ^{3}(\omega t) \mathrm{d} t
$$

where $T$ is the period of the harmonic oscillation. Substituting equations (B.1) and (B.2), this becomes

$$
\bar{P}_{w}=A_{i} \rho \frac{U^{3}}{\pi C_{v}^{2} \Phi^{2}} \int_{0}^{1}\left(1-y^{2}\right) \mathrm{d} y=A_{i} \rho \frac{2 U^{3}}{3 \pi C_{v}^{2} \Phi^{2}} .
$$

One can define the time-averaged non-linear plate resistance as $R_{\text {plate,NL, } t}$ using the expression

$$
\bar{P}_{w}=\frac{1}{2} A_{i} R_{\mathrm{plate}, \mathrm{NL}, t} U^{2}
$$

This implies that

$$
R_{\mathrm{plate}, \mathrm{NL}, t}=\frac{4 \rho U}{3 \pi C_{v}^{2} \Phi^{2}} .
$$

Using as reference the expression in Temiz et al. [8] $\left(R_{\text {ref }}=(\rho U) /\left(2 C_{v}^{2} \Phi^{2}\right)\right)$ one finds the theoretical asymptote:

$$
\frac{R_{\mathrm{plate}, \mathrm{NL}, t}}{R_{\mathrm{ref}}}=\frac{8}{3 \pi}=0.849 .
$$

The value of $R_{\mathrm{plate}, \mathrm{NL}, t}$ is used to derive the analytical asymptote in Figures $8-10$. This value is fairly close to the asymptote $0.7<R_{\text {plate,NL }, t} / R_{\text {ref }}<0.8$ found in Temiz et al. [6] and Auriemma [8]. Temiz et al. [8] is considering circular perforations with sharp square edges. Auriemma is considering slit shaped perforations with right angled edges. In the geometry proposed in this work, the channel length is zero.

\section{Appendix C}

\section{C.1 Correction for boundary layer thickness}

For $\hat{p}\left(x_{\text {ref }}\right)=40 \mathrm{~Pa}$ (really high amplitude) and $C_{v}=$ 0.82 , we have $\left|\hat{u}_{p}\right|=C_{v} \sqrt{\frac{2 \Delta \hat{p}}{\rho}}=6.7 \mathrm{~m} / \mathrm{s}$. For $b=0.5 \mathrm{~mm}$, the Reynolds number $\operatorname{Re}_{b}=\rho U_{p} b / \mu=223$. The steady viscous boundary layer has a thickness of the order of $\delta_{v} / b \approx \sqrt{\frac{1}{R e_{b}}} \approx 0.1$. Therefore, one can expect the frictionless theory to have an accuracy of the order of $10 \%$. For very high amplitudes, using the Twaites solution [35] of the integral boundary layer equation of Von Karman one can obtain an estimation of the viscous boundary layer thickness. One has

$$
\theta^{2} U_{p}^{6} \approx 0.45 v \int_{-t}^{0}[U(x)]^{5} \mathrm{~d} x
$$

with $\theta=\int_{0}^{\delta_{v}} \frac{y}{\delta_{v}}\left(1-\frac{y}{\delta_{v}}\right) \mathrm{d} y$ is the momentum thickness of the viscous boundary layer of thickness $\delta_{v}$ and $x=0$ is at the slit neck. Neglecting the boundary layer thickness and assuming a uniform velocity $U(x)$ the mass conservation law becomes

$$
U(x)(b-2 x) \approx U_{p} b
$$

because the slit angle is $\pi / 2$ (Fig. A.1) and for $t \gg b$

$$
\frac{\theta^{2}}{b^{2}} \approx \frac{0.45 v}{U_{p} b^{2}} \int_{-t}^{0} \frac{1}{\left(1-2 \frac{x}{b}\right)^{5}} \mathrm{~d} x \approx \frac{1}{8} \frac{0.45 v}{U_{p} b}
$$


Assuming a linear velocity profile in the boundary layer of thickness $\delta_{v}$, one has a displacement thickness $\delta_{v}^{*}=\delta_{v} / 2$ and a momentum thickness $\theta=\delta_{v} / 6$. This implies:

$$
\frac{\delta_{v}^{*}}{b}=\approx \frac{3 \theta}{b} \approx \sqrt{\frac{9}{8} \frac{0.45 v}{U_{p} b}} .
$$

Given $\operatorname{Re}_{b}=\rho b U_{p} / \mu \approx 223$ one has $\delta_{v}^{*} \approx 0.05 b$. This implies a reduction of the power because of the reduction of the porosity $\Phi_{\text {eff }}=\Phi\left(1-2 \delta_{v}^{*} / b\right)$ so that

$$
\frac{R_{\text {plate, } \mathrm{NL}, t}}{R_{\mathrm{ref}}}=\frac{\Phi^{2}}{\Phi_{\text {eff }}^{2}} \frac{8}{3 \pi} \approx 1.05 \text {. }
$$

